\title{
Predicting future ecological degradation based on modelled thresholds
}

\author{
Peter G. Fairweather*, Rebecca E. Lester \\ School of Biological Sciences, Flinders University, GPO Box 2100, Adelaide, South Australia 5001, Australia
}

\begin{abstract}
Threshold models are becoming important in determining the ecological consequences of our actions within the environment and have a key role in setting bounds on targets used by natural resource managers. We have been using thresholds and related concepts adapted from the multiple stable-states literature to model ecosystem response in the Coorong, the estuary for Australia's largest river. Our modelling approach is based upon developing a state-and-transition model, with the states defined by the biota and the transitions defined by a classification and regression tree (CART) analysis of the environmental data for the region. Here we explore the behaviour of thresholds within that model. Managers tend to plan for a set of often arbitrarily-derived thresholds in their natural resource management. We attempt to assess how the precision afforded by analyses such as CART translates into ecological outcomes, and explicitly trial several approaches to understanding thresholds and transitions in our model and how they might be relevant for management. We conclude that the most promising approach would be a mixture of further modelling (using past behaviour to predict future degradation) in conjunction with targeted experiments to confirm the results. Our case study of the Coorong is further developed, particularly for the modelling stages of the protocol, to provide recommendations to improve natural resource management strategies that are currently in use.
\end{abstract}

KEY WORDS: Coorong · Ecosystem states · Empirical anticipation - Environmental futures · Physico-chemical transitions $\cdot$ South Australia $\cdot$ Statistical modelling $\cdot$ Water allocation

\section{INTRODUCTION}

The management of coastal marine ecosystems such as estuaries is becoming increasingly based upon a mixture of empirical field monitoring and modelling of possible future ecological conditions. The importance of identifying thresholds for management is now more apparent than ever (Scheffer 2009, Suding \& Hobbs 2009). The existence of such thresholds may be indicated by abrupt transitions from one ecosystem state to another, thus displaying regime shifts (deYoung et al. 2008) or catastrophic behaviour (Folke et al. 2004). These transitions are assumed to occur when the underlying physicochemical drivers within an ecosystem exceed a certain value (the threshold) that flips the system into a new, alternative state. Awareness of the possibility of these sudden transitions, including their implications for management, is currently high (e.g. van de Koppel et al. 2001,
Lozano-Montes et al. 2008, Andersen et al. 2009, Scheffer et al. 2009). Searching for these thresholds involves detecting response to future climate change (Dakos et al. 2008, Scheffer 2009) or dire outcomes like benthic hypoxia (Conley et al. 2009) or coral reef loss (Mumby et al. 2007) that managers wish to avoid.

Current understanding of the role of thresholds is often based upon conceptual models of how the systems work (Dennison et al. 2007), in particular the key processes that give an ecosystem its distinctive character that is valued by the public. Many estuaries and coastal wetlands derive much of this character from the flows of fresh water coming downstream from their associated rivers. Excessive extraction of water upstream for human uses can, in turn, threaten an estuary's future and considerable progress has been made recently in modelling these relationships using relatively simple models (e.g. Kim \& Montagna 2009, Lester \& Fair- 
weather 2009a,b, 2010, Zweig \& Kitchens 2009). Many of these modelling approaches, such as state-and-transition modelling, have implied thresholds associated with whatever environmental changes trigger the transitions between identified states. Especially of interest is how likely future climate change might influence flow regimes and consequential ecological effects (e.g. Powell 2008, Lester et al. 2009). These issues have long been recognised in freshwater ecology, where flows determine many aspects of ecosystem behaviour (Anderson et al. 2006), but the challenges of anticipating rare or extreme events are less routinely considered in marine ecology (but see Fuentes et al. 2006, Denny et al. 2009, Kimmel et al. 2009), despite the predicted increase of these events under climate change and their potential role in shaping ecosystems. The search for early-warning signals of thresholds is still in its infancy (Andersen et al. 2009, Scheffer et al. 2009), and so we believe that a variety of approaches needs to be explored and critically evaluated.

There is an inherent difficulty in predicting thresholds that are approaching but not yet apparent, but this is precisely what needs to occur to effectively manage non-linear systems. Previous work on the detection of upcoming transitions has focused upon recognising increasing variance of response variables as a threshold was approached (Carpenter \& Brock 2006), shifts in spectral properties of time series near a threshold (Kleinen et al. 2003) or a slowing in rates of change (Dakos et al. 2008). Thus the focus is often upon the further moments of distributions of some variables (as opposed to just their means or central tendencies), where scientists become alert to increased overall variability within assemblages (Warwick \& Clarke 1993) or changes in skewness (Guttal \& Jayaprakash 2008). These properties have been demonstrated using deterministic models of response variables (e.g. Mumby et al. 2007, Carpenter et al. 2008) and, while threshold dynamics have been found to follow theoretical expectations, changes in many of these parameters have not been detected in simulated data prior to the threshold being crossed (e.g. see Carpenter et al. 2008). This means that they may be of limited use in predicting future transitions from measured or simulated time series of driving variables.

Over the last $10 \mathrm{yr}$, there has been a major decline in the ecological condition of the Coorong, the estuary and terminal lagoon system for Australia's largest river system, the River Murray (Brookes et al. 2009). This decline is due to prolonged drought combined with past management of the Murray-Darling Basin. In order to successfully manage these lagoons in the future, predictions are needed to evaluate the effects of possible management actions on the Coorong ecosystems under a variety of climatic scenarios. To pro- vide such predictions, thresholds and concepts derived from the multiple stable-states literature have been used to model ecosystem response in the Coorong (Lester \& Fairweather 2009b, 2010). The model itself is a state-and-transition model, with the states defined by the biotic assemblages found, and the transitions defined by a classification and regression tree (CART) analysis (De'ath \& Fabricius 2000) of the physicochemical data for the region (Lester \& Fairweather 2009a,b, 2010). Thus these transitions between ecosystem states define the thresholds of interest for the Coorong, and can be used by managers to set objectives to manage towards or to define the limits of acceptable change.

We have been developing an understanding of thresholds for these ecosystem states in the Coorong lagoons subsequent to our initial modelling approach. The sensitivity of the ecosystem to and the behaviour near these thresholds is, however, currently unknown. Managers (in Australia at least) tend to manage to an often arbitrarily-defined set of limits in natural resource management. Here we explore how thresholds identified by analyses such as CART could be used to provide advance warning of upcoming ecological shifts. We use the Coorong model as an example to outline a more general protocol for assessing the utility of thresholds to derive leading indicators, focus upon modelling the states via that protocol and provide recommendations regarding targeted experiments to confirm our findings aimed at improving the natural resource management strategies currently in use.

A better understanding of these thresholds would aid managers in determining which changes within their dynamic system are worthy of concern. It would thus allow them to target monitoring to better detect when to act, and also fine-tune their actions based upon the outcomes of that monitoring. Here we explored threshold dynamics based on an existing ecological response model to identify whether any could be of assistance to managers. Thus the aims of the present study were to (1) describe the threshold dynamics within the ecosystem states model for the Coorong, with a view to predicting upcoming switches in state, and (2) evaluate a variety of approaches for how to assess which changes matter, and especially their utility for an early-warning capacity as leading indicators of ensuing change.

\section{METHODS}

Study area. The Coorong is the estuary at the terminus of the River Murray, the largest river system in Australia. The estuary is part of a Ramsar Conventionlisted Wetland of International Importance, and pro- 
vides important breeding and feeding habitat for many species of birds and fish (Brookes et al. 2009). The Coorong is a long, shallow lagoonal system, which is typified by a natural gradient in environmental conditions from an estuary around the Murray Mouth to hypersaline conditions at the southern, terminal end of the system, more than $100 \mathrm{~km}$ away. The Coorong can thus be divided into 3 regions: the usually estuarine Murray Mouth region at the northern end; and 2 lagoons separated by a constriction, the North and South Lagoons. River Murray flows can be regulated across a system of barrages at the northern end, with much smaller volumes entering the South Lagoon at Salt Creek, the Coorong's only tributary. Additional information on the characteristics of the system, including maps, are presented in Brookes et al. (2009) and Lester \& Fairweather (2009b, 2010).

Ecosystem state model. An ecosystem response model was developed for the Coorong based on ecosystem states (Lester \& Fairweather 2009a,b, 2010). The model is described in detail elsewhere (e.g. Lester \& Fairweather 2009a) but is summarised to provide context for the further analyses of threshold dynamics presented here. The ecosystem states approach identifies clusters of biota that occur together in space and time and then finds thresholds for physico-chemical parameters that are associated with the presence of each cluster. Briefly, 2 data sets were compiled of the available biological and physico-chemical (or environmental) data, respectively. The biological data included survey data for fish, birds, benthic macroinvertebrates and macrophytes across 12 sites between 1999 and 2007. The environmental data included water quality and quantity, flow and meteorological variables over the same time period. Particular atten- tion was given to including maxima, minima, lagged values and variances in the environmental data set (thus 230 variables in total), because mean variables are not always the best predictors of biological communities (Gaines \& Denny 1993). The provenance of the data sets used and references to the collection methods are presented in Lester \& Fairweather (2009b).

Clusters of co-occurring biota (including macrophytes, birds, fish and benthic macroinvertebrates) in space and time were identified from the biological data set using group-average clustering in PRIMER v6.0 (Clarke \& Gorley 2006). Cluster membership was then used as the response variable for a CART analysis performed in CART Pro v6.0 (Steinberg \& Golovnya 2007). CART analysis sequentially splits a response variable to maximise the difference between pre-defined groups by identifying thresholds (or splitting points) in the best of a range of independent variables (here the environmental data set) (Steinberg \& Golovnya 2007). Thus the environmental conditions that were associated with each of the biotic clusters were identified. The biological data from cases in each CART terminal node were then tested for biological distinctness using analysis of similarities in PRIMER v6.0 (Clarke \& Gorley 2006) and were combined when not statistically different. Predictive capacity was tested using cases withheld from the original model development and each group (or ecosystem state) was characterised based on its biological and environmental properties (e.g. using similarity percentage in PRIMER v6.0; Clarke \& Gorley 2006).

For the Coorong, 8 ecosystem states were identified. Associated triggers in terms of physico-chemical parameters (with their relevant threshold values given in Table 1) were identified as average daily tidal range,

Table 1. The sequence of thresholds that determine each ecosystem state (see Lester \& Fairweather 2009a, 2010 for a diagrammatic representation of the model and species lists for each state). A transition in ecosystem state will occur whenever one of the thresholds relevant for the original ecosystem state is crossed (as indicated by horizontal lines). Two horizontal lines are given for the maximum number of days since flow because that threshold appears in the model twice, '-' indicates that a threshold is not relevant for the ecosystem state in question. For example, no transition will occur from the Estuarine/Marine state if the water level, depth or salinity thresholds are crossed. Depth is from the previous year, consistent with the predictive variable identified in the ecosystem state model (refer to text for additional information). m AHD: metres above the Australian Height Datum, which approximates sea level

\begin{tabular}{|c|c|c|c|c|c|c|c|}
\hline Ecosystem state & $\begin{array}{c}\text { Relative } \\
\text { state health }\end{array}$ & $\begin{array}{l}\text { Tidal range } \\
\qquad(\mathrm{m})\end{array}$ & $\begin{array}{l}\text { Maximum no. } \\
\text { days since } \\
\text { flow }\end{array}$ & $\begin{array}{c}\text { Water } \\
\text { level A } \\
\text { (m AHD) }\end{array}$ & $\begin{array}{c}\text { Water } \\
\text { level B } \\
\text { (m AHD) }\end{array}$ & $\begin{array}{l}\text { Depth } \\
\text { (m) }\end{array}$ & $\begin{array}{c}\text { Salinity } \\
\left(\mathrm{g} \mathrm{l}^{-1}\right)\end{array}$ \\
\hline Estuarine/Marine & Healthy & $>0.05$ & $\leq 339$ & - & - & - & - \\
\hline Marine & Degraded & $>0.05$ & $>339$ & - & - & $>1.99$ & - \\
\hline Unhealthy Marine & Degraded & $>0.05$ & $>339$ & - & - & $\leq 1.99$ & $\leq 64.5$ \\
\hline Degraded Marine & Degraded & $>0.05$ & $>339$ & - & - & $\leq 1.99$ & $>64.5$ \\
\hline Healthy Hypersaline & Healthy & $\leq 0.05$ & $\leq 339$ & $>0.37$ & - & - & - \\
\hline Average Hypersaline & Healthy & $\leq 0.05$ & $\leq 339$ & $\leq 0.37$ & - & - & - \\
\hline Unhealthy Hypersaline & Degraded & $\leq 0.05$ & $>339$ & - & $>-0.09$ & - & - \\
\hline Degraded Hypersaline & Degraded & $\leq 0.05$ & $>339$ & - & $\leq-0.09$ & - & - \\
\hline
\end{tabular}


the maximum number of days without barrage flows, 2 average annual water levels, average annual depth from the previous year (thus a temporal component was important here that was not so for other predictive variables) and average annual salinity. Various sequential combinations of parameter values for each of these 6 thresholds combine (Table 1) to predict the ecosystem state for a given case (note that the threshold for maximum number of days without flow of 339 appears twice in the model-once for cases with an average daily tidal range above the threshold, and once for those below the threshold - hence there are 6 thresholds that specify one of 8 possible states, rather than the 7 that would usually be required; see Lester \& Fairweather 2009a,b for the model).

Because of the sequential nature of the thresholds in the ecosystem states model (a characteristic of the CART models upon which it is based; De'ath \& Fabricius 2000), not all crossings of a threshold result in a change in ecosystem state. For example, changes in average annual salinity over the threshold do not result in ecosystem state changes unless depth from the previous year is less than $1.99 \mathrm{~m}$, the maximum number of days since flow is greater than 339 and the average daily tidal range is greater than $0.05 \mathrm{~m}$ (Table 1 ). Table 1 illustrates this sequence of thresholds and allows the reader to determine when crossing a particular threshold will result in a change of state. In considering the threshold dynamics of the model, we have investigated both crossings of the threshold (whether or not an ecosystem-state change occurred) and only those crossings that resulted in a change in state (transitions), but have focused on the latter as most relevant to the management of the system.

Of the 8 possible ecosystem states, 5 occurred within the Coorong only when the threshold for the maximum number of days without barrage flow was exceeded. Based on modelled natural flows for the Murray-Darling Basin, end-of-system flows down the River Murray cease in only $1 \%$ of years (CSIRO 2008), so we defined these 5 ecosystem states as being degraded. We believe that it is justified to consider a period of more than 11 mo without end-of-system flows as a degraded condition for the Coorong, and this is supported by the relatively simplified biotic assemblages associated with those 5 ecosystem states (see Lester \& Fairweather 2009a,b for characteristics and species lists typical of each ecosystem state).

Each of the ecosystem states has been characterised based on its biological characteristics. As an example, the Average Hypersaline state (one of the healthy states lacking a tidal influence) was characterised by relatively low numbers of fish, including greenback flounder Rhombosolea tapirina and mulloway Argyrosomus japonicus. Correspondingly, few piscivorous bird species were associated with the state (with the exception of the Australian pelican Pelecanus conspicillatus). Instead, bird communities included waders (e.g. banded stilt Cladorhynchus leucocephalus, red-necked stint Calidris ruficollis and red-necked avocet Recurvirostra novaehollandiae) and waterfowl (e.g. grey teal Anas gracilis). Ruppia tuberosa, a macrophyte, had greater coverage here than for any other state for which data was available. There were relatively few benthic invertebrate taxa associated with this state, but chironomid larvae and amphipods occurred in high numbers. This state had moderate average salinities and low average depths, but freshwater flows occurred regularly. Water quality characteristics included high nutrient concentrations (e.g. total Kjeldahl nitrogen and ammonia), chlorophyll $a$ and $b$ concentrations and turbidity. In contrast, the Unhealthy Hypersaline state had higher average salinities, low average water levels and low variability in water levels with long periods since freshwater flows. Nutrient concentrations and turbidity remained high. Smallmouthed hardyhead Atherinosoma microstoma was the only fish species present in any large numbers. Avifauna were characterised by hoary-headed grebe Poliocephalus poliocephalus, Australian pelican (both piscivorous), banded stilt (a wader) and Australian shelduck Tadorna tadornoides (a waterfowl). This state supported a very limited diversity of invertebrates including polychaetes, but still had high numbers of chironomid larvae. Similar descriptions of the remaining states, along with tabulated average values for each, are presented in Lester \& Fairweather (2009a).

Baseline scenario for the Coorong. In order to predict a sequence of ecosystem states for the region, the ecosystem states model was used in combination with a climate model, a river model and a hydrodynamic model for the region (Webster 2007, CSIRO 2008). A sequence of daily flows for the Coorong was developed using historical climate data with current infrastructure and extraction levels for the Murray-Darling Basin for the period of 1895 to 2008, largely in line with Scenario A in the Murray-Darling Basin Sustainable Yield Project (CSIRO 2008). This, coupled with average daily flows from the south via the Salt Creek tributary (from 2001 to 2008) and sea levels in Encounter Bay, was used as input to a hydrodynamic model developed for the Coorong (Webster 2007, Lester et al. 2009). The hydrodynamic model then predicted hourly water levels and salinities along the length of the Coorong over the 114 yr model run that were used as input to the ecosystem states model. The ecosystem states model predicted an annual time series of ecosystem states for each of 12 focal sites spread along the Coorong (see Lester \& Fairweather 2009a, 2010 for additional detail). Thus the model predicted ecosystem states for a hypothetical 114 yr sequence that had historical climate 
variability but present-day infrastructure and extraction levels throughout. Such a sequence provides an understanding of the level of variability in ecosystem condition under a particular climate scenario, rather than attempting to replicate historical conditions exactly.

Threshold analyses. Some basic statistics were calculated for each threshold. These included the number of times each threshold was crossed under the baseline scenario simulation (described above), the return interval for crossing each threshold and the average number of years for which that threshold was exceeded (average exceedance interval).

The specific sites at which thresholds were crossed and the years in which each crossing occurred were also identified. Years in which thresholds were crossed at each site were identified using the R statistical environment v2.8.1 (R Core Development Team 2009). The years in which thresholds were crossed were also compared with lists of El Niño and La Niña years in Australia (BOM 2009). The El Niño Southern Oscillation (ENSO) affects large-scale Australian weather patterns, with drought periods associated with El Niño years and wetter periods with La Niña years. This pattern is in contrast to the associations seen in other parts of the world.

Determining rates of change: It is feasible that physico-chemical time series might exhibit different behaviour when they approach a threshold. Thus we wanted to see whether we could predict upcoming thresholds based on something simple, like changes in rates of change over time. Previous research on response variables suggests that rates of change slow as a threshold is approached (Dakos et al. 2008), and this may also be the case for predictive variables. A series of nested rates of change were developed for various time periods preceding each unique crossing of each threshold. For each site, rates of change were calculated for the week, month, year and decade prior to each threshold crossing. In order to preserve statistical independence, where a particular threshold was crossed at more than one site per year, only one site was selected at random for inclusion in the analysis. The site to be included was selected post hoc using a random number generator (having assigned each site a numeric tag). This site was included in the analysis and all other sites where the thresholds were crossed in the same year were excluded. Absolute values were taken for each rate of change, and the minimum, maximum and mean rates of change were calculated for each time step. Rates of change were calculated for each time period using linear regression in SYSTAT v.11 (SYSTAT 2004). Standardised rates of change were also computed by dividing each data point by the maximum rate of change observed within that time series.
This scaling allowed comparison of trends for variables changing over different ranges. Confidence intervals were calculated for each rate of change (and standardised rate of change) by bootstrapping the mean rate of change using the 'boot' function in R.

Characterising transitions: Years in which individual sites crossed each threshold in the ecosystem state model were identified in the baseline scenario described above. For each, the value of the relevant parameter was recorded for the year in which the transition occurred and also for the following year to understand what values each variable takes immediately on either side of a threshold crossing.

Logistic regressions: Understanding the width of any transition zone for predictive variables around a threshold could assist in predicting whether upcoming transitions are likely. If these transition zones are sufficiently narrow, when values fall within that zone may be a good indication that a transition is imminent. Logistic regression is a classic method for exploring the shape of changes between binary variables, such as 2 ecosystem states governed by a threshold. Logistic regression was used for those thresholds that were crossed enough times to make analysis meaningful (here, a cut-off of more than 20 times over the $114 \mathrm{yr}$ of the baseline scenario was used; see 'Results' for the number of times that each transition occurred), using the values that these physico-chemical variables took immediately prior to and following each transition. Binomial general linear models were fitted using the bias-correction algorithm for maximum likelihood estimates developed by Firth (1993). This was done using the 'brglm' package developed for R (Kosmidis 2007).

Zones of transition: Another method for exploring the width of the transition zone is to explicitly investigate the range of values that variables take immediately before and after a transition. Again, this could potentially assist in identifying a window within which transition is likely, where driving variables falling within the range could prompt management intervention. Again, this window would need to be sufficiently narrow to be of value for management. Maxima and minima were identified both before and after each threshold was crossed in each year in which it was crossed and the extremes of these were used to derive a zone of transition. This zone of transition comprised the minimum value of the parameter of interest in the year that threshold was exceeded and the maximum value of the same parameter the year after (and vice versa for falling below a threshold). As such, it gave the maximum range of values observed on either side of a threshold crossing. For each site, and for the Coorong as a whole, the proportion of time in which predicted parameter values fell within this zone of transition was calculated. 
Developing a predictive model for future degraded ecosystem states: Identifying an appropriate time lag: An attempt was made to predict future degradation by comparing the ecosystem state in any year to physicochemical conditions several years prior. Thus choice of the time lags to include was a key step. Cross-correlation analyses were used to identify significant time lags between pairs of flow, hydrodynamic and biological variables. These analyses were conducted using the cross-correlation plotting function in the time series module of SYSTAT v.11 (SYSTAT 2004). Based on these cross-correlations, tables were compiled to summarise the significant time lags identified.

A subset of the 230! (factorial, thus effectively infinite) possible combinations of cross-correlations were undertaken (with 230 being the total number of environmental variables used in the original model development; refer to the 'Ecosystem state model' section above). This subset was chosen based on our understanding of the likely causal relationships within the system, as well as relationships that were identified in previous analyses (e.g. see Lester \& Fairweather 2009a). We focused upon 4 types of correlations: correlations among flow variables (pertaining directly to the amount of flow passing over the barrages, e.g. total flow volume); correlations between flow and hydrodynamic variables (variables that were outputs from the hydrodynamic model, e.g. water levels and salinities); correlations between flow or hydrodynamic and biological variables; and correlations among biological variables.

For flow, total flow volume per annum and average daily flow volume were identified as the parameters likely to be correlated with the hydrodynamic variables for the Coorong. The specific hydrodynamic variables investigated included the average, maximum, minimum and variance of water levels and salinity for each of the North and South Lagoons. These were selected because they are easily measured, interpretable and likely to be of significance to the biotic assemblages in the region.

Biological variables investigated were chosen as a representative subset of the assemblage present, from the data used in the construction of the ecosystem state model. The species selected were chosen either because of identified ecological significance (e.g. Ruppia tuberosa has been previously identified as an ecosystem engineer in the region; Rogers \& Paton 2009), or because they had previously been identified as typical of one or more ecosystem states (Lester \& Fairweather 2009a). Biological variables included the proportion of sediment cores containing $R$. tuberosa (as a measure of the coverage of that macrophyte); abundances of a selection of bird species, including 2 wader species (red-necked stint and banded stilt), 2 piscivorous species (little pied cormorant Phalacrocorax melanoleucos and Australian pelican) and 2 waterfowl (grey teal and Australasian shelduck); catch per unit effort for 3 commercial fish species (Australian salmon Arripis truttaceus, black bream Acanthopagrus butcheri and yellow-eyed mullet Aldrichetta forsteri); and abundances of 3 benthic macroinvertebrate groups (chironomid larvae and the polychaetes Australonereis ehlersi and Capitella sp.).

The year with the single strongest correlation (whether positive or negative) was identified for each cross-correlation comparison, separating flow correlated with hydrodynamic variables from flow or hydrodynamic variables correlated with biological variables. From this analysis, those years that had the strongest correlations most often were identified as time lags to be used in the predictive modelling step.

Modelling future degraded ecosystem states for chosen time lags: The most significant time lags identified with cross-correlations were retained for use in predicting future degraded ecosystem states. Where several years had very similar numbers of significant cross-correlations (Table 2), time lags were chosen in order to allow sufficient time for management intervention (i.e. a 3 yr time lag) and to investigate how the predictors of future degraded ecosystem states changed over shorter times (i.e. a 1 yr lag).

Ecosystem states were modelled for 12 sites along the length of the Coorong (see Lester \& Fairweather 2009b for details) for the period of 1999 to 2007 using

Table 2. Numbers of significant time lags among flow, hydrodynamic and biological cross-correlations. Bold numbers indicate the highest number of statistically significant correlations for each column. For flow versus hydrodynamic variables, $n=19$. For biological versus flow, hydrodynamic or biological variables, $n=82$. Biological variables were cross-correlated against a combination of flow and hydrodynamic variables, as well as among biological variables. Refer to 'Methods: Identifying an appropriate time lag' for additional information on the variables used

\begin{tabular}{|c|c|c|c|c|c|c|}
\hline \multirow[t]{2}{*}{$\begin{array}{l}\text { Time lag } \\
(\mathrm{yr})\end{array}$} & \multicolumn{3}{|c|}{$\begin{array}{l}\text { Flow vs. hydrodynamic } \\
\text { variables }\end{array}$} & \multicolumn{3}{|c|}{$\begin{array}{l}\text { Biological vs. flow, hydrodynamic } \\
\text { or biological variables }\end{array}$} \\
\hline & Positive & Negative & Total & Positive & Negative & Total \\
\hline 1 & 9 & 9 & 18 & 5 & 2 & 7 \\
\hline 2 & 7 & 7 & 14 & 4 & 4 & 8 \\
\hline 3 & 8 & 6 & 14 & 3 & 3 & 6 \\
\hline 4 & 10 & 4 & 14 & 3 & 3 & 6 \\
\hline 5 & 8 & 0 & 8 & 4 & 3 & 7 \\
\hline 6 & 2 & 6 & 8 & 4 & 3 & 7 \\
\hline 7 & 0 & 3 & 3 & 5 & 1 & 6 \\
\hline 8 & 0 & 9 & 9 & 5 & 2 & 7 \\
\hline 9 & 8 & 7 & 15 & 2 & 2 & 4 \\
\hline
\end{tabular}


the model described in Lester \& Fairweather (2009a,b, 2010), with each site for each year referred to as a siteyear. These predicted ecosystem states were offset from the accompanying physico-chemical data set by each of the identified significant lag periods (i.e. either 1 or 3 yr). The physico-chemical data set used was similar to that described above and in Lester \& Fairweather (2009a,b, 2010), and so included a range of flows, water levels, depths, salinities, meteorological variables and water quality variables described by means, maxima, minima and variances. In addition, variables averaging the hydrodynamic conditions across all sites in each of the 2 lagoons were added to the list. Averaged variables were included to represent what is already in use by managers. These included maxima, minima, means and variances of water levels and salinities for each of the North and South Lagoons.

The ecosystem states were then classified as either degraded or healthy (i.e. not-degraded) based on whether there had been freshwater input from the River Murray in the previous 339 d (as described above). CART analyses were used to identify specific physico-chemical variables associated with the presence of future degraded ecosystem states. The analyses were conducted in CART v6.0 (Steinberg \& Golovnya 2007) using a twoing-splitting algorithm and 10 -fold cross-validation. The minimum size of a parent node was set to 5 , with the minimum size of a child node at 2 . The best tree was selected using the 1-standard error rule (Breiman et al. 1984), and variables were penalised for missing values using a value of $\beta=$ 0.60 (Steinberg \& Golovnya 2007). Pearson correlations between variables identified as significant pre- dictors of future degraded ecosystem states were analysed using linear regression and, where significant correlations ( $\alpha=0.05)$ among predictor variables existed, the variable with the lower level of importance for the model was excluded and the model then re-run. This approach was consistent with that used originally to construct the ecosystem state model.

\section{RESULTS}

\section{Threshold characteristics}

Within the model simulation, the system crossed the different thresholds with uneven frequencies and crossing events were not distributed equally across all sites (Table 3). The return interval and duration of exceedance of thresholds were not equal amongst the 3 regions within the Coorong (Table 3), but were inversely related. The thresholds involving salinity, tidal range and water level A were most commonly observed to be crossed (Table 3), whereas the other thresholds were crossed either uncommonly (maximum number of days since flow, depth) or only once (water level B). Water level A and tidal-range thresholds were crossed more commonly at the northern end of the Coorong (i.e. the Murray Mouth or North Lagoon regions), while the salinity threshold was crossed more frequently in the South Lagoon.

There was no obvious relationship between threshold crossings and the ENSO index. All variables were crossed most frequently in years that were not designated as either El Niño or La Niña years

Table 3. Summary of threshold characteristics. These values are based on a simulation of historical conditions within the Coorong over 114 yr. The coincidence with El Niño Southern Oscillation (ENSO) columns illustrate which threshold crossings coincided with El Niño years, La Niña years or neither. The return interval and exceedance durations show the average number of years between threshold crossings and the average length of time that a site remained over a threshold, respectively, for each of the 3 regions (Murray Mouth [MM], North Lagoon [NL] and South Lagoon [SL]). Depth is from the previous year, consistent with the predictive variable identified in the ecosystem state model (refer 'Methods: Ecosystem state model' for additional information). The depth threshold was only crossed at North Lagoon sites. The number of days since flow occurred over the barrages is not spatially variable, so the return intervals and exceedance durations presented under the MM column are representative of the whole Coorong. The water level B threshold was only crossed in the final year of simulation for the 3 southernmost sites (SL), so it was not possible to calculate return intervals or exceedance duration. na: no value possible; m AHD: metres above the Australian Height Datum, which approximates sea level

\begin{tabular}{|c|c|c|c|c|c|c|c|c|c|c|c|}
\hline \multirow[t]{2}{*}{ Threshold } & \multirow{2}{*}{$\begin{array}{l}\text { Threshold } \\
\text { value }\end{array}$} & \multirow{2}{*}{$\begin{array}{l}\text { No. years } \\
\text { crossed }\end{array}$} & \multicolumn{3}{|c|}{ Coincidence ENSO (yr) } & \multicolumn{3}{|c|}{ Return interval (yr) } & \multicolumn{3}{|c|}{ Exceedance duration (yr) } \\
\hline & & & El Niño & La Niña & Neither & MM & NL & SL & MM & NL & SL \\
\hline Tidal range & $0.05 \mathrm{~m}$ & 18 & 6 & 2 & 10 & na & 2.6 & 20.5 & na & 13.8 & 4.6 \\
\hline $\begin{array}{l}\text { Maximum no. } \\
\text { days since flow }\end{array}$ & $339 \mathrm{~d}$ & 4 & 3 & 0 & 1 & 34.3 & na & na & 1.8 & na & na \\
\hline Salinity & $64.5 \mathrm{~g} \mathrm{l}^{-1}$ & 24 & 9 & 2 & 13 & na & 10.3 & 5.6 & na & 7.3 & 9.1 \\
\hline Depth & $1.99 \mathrm{~m}$ & 6 & 0 & 3 & 3 & na & 15.0 & na & na & 1.7 & na \\
\hline Water level A & $0.37 \mathrm{~m}$ AHD & 11 & 2 & 3 & 6 & 8.2 & 5.0 & 10.2 & 1.8 & 1.6 & 1.6 \\
\hline Water level B & $-0.09 \mathrm{~m} \mathrm{AHD}$ & 1 & 0 & 0 & 1 & na & na & na & na & na & na \\
\hline
\end{tabular}


(including when the relative proportion of ENSO years was taken into account), so we identified no direct link between thresholds and ENSO in the present study.

\section{Determining rates of change}

The rate of change in predictive variables increased as a threshold was approached for those thresholds where analysis was possible. This included salinity, water level A, tidal range and depth. Analysis of a rate of change is nonsensical for a count variable such as the number of days since flow, so that was not attempted. The threshold for water level B was only crossed in a single year, so it had an insufficient sample size for analysis. Differences in the scale of variables somewhat masked the increasing rate of change for the tidal range, water level A and depth thresholds (Fig. 1a vs. Fig. 1b), but all tended to increase within $1 \mathrm{mo}$ of the transition, or $1 \mathrm{yr}$ for the salinity threshold (and depth to a lesser extent; see Fig. 1b). The patterns illustrated in Fig. 1 are for mean rates of change, but minimum and maximum rates of change also exhibited the same pattern. In addition to increasing rates of change near a transition, the bootstrapped variability of the rate of change also increased as a transition approached (Fig. 1), again becoming apparent approximately 1 mo before the transition occurred.

\section{Characterising transitions}

Transitions between ecosystem states occurred less frequently than threshold crossings (as defined above). Transitions between marine states and hypersaline states occurred 61 times over the $114 \mathrm{yr}$ model run. Transitions over the maximum number of days since flow threshold for marine states occurred 51 times (i.e. from Estuarine/Marine to Marine, Unhealthy Marine or Degraded Marine states; see Table 1), while transitions over the same threshold occurred 23 times for hypersaline states (i.e. from Healthy Hypersaline or Average Hypersaline to Unhealthy Hypersaline or Degraded Hypersaline; see Table 1 ). There were only 3 transitions observed between the Unhealthy Marine and Degraded Marine states (salinity threshold), 3 between Unhealthy Hypersaline and Degraded Hypersaline states (water level B threshold) and none between the Marine and either the Unhealthy or Degraded Marine states (depth threshold). The remaining results will relate to transitions between states, rather than the crossing of individual thresholds (which may or may not result in a shift in ecosystem state).
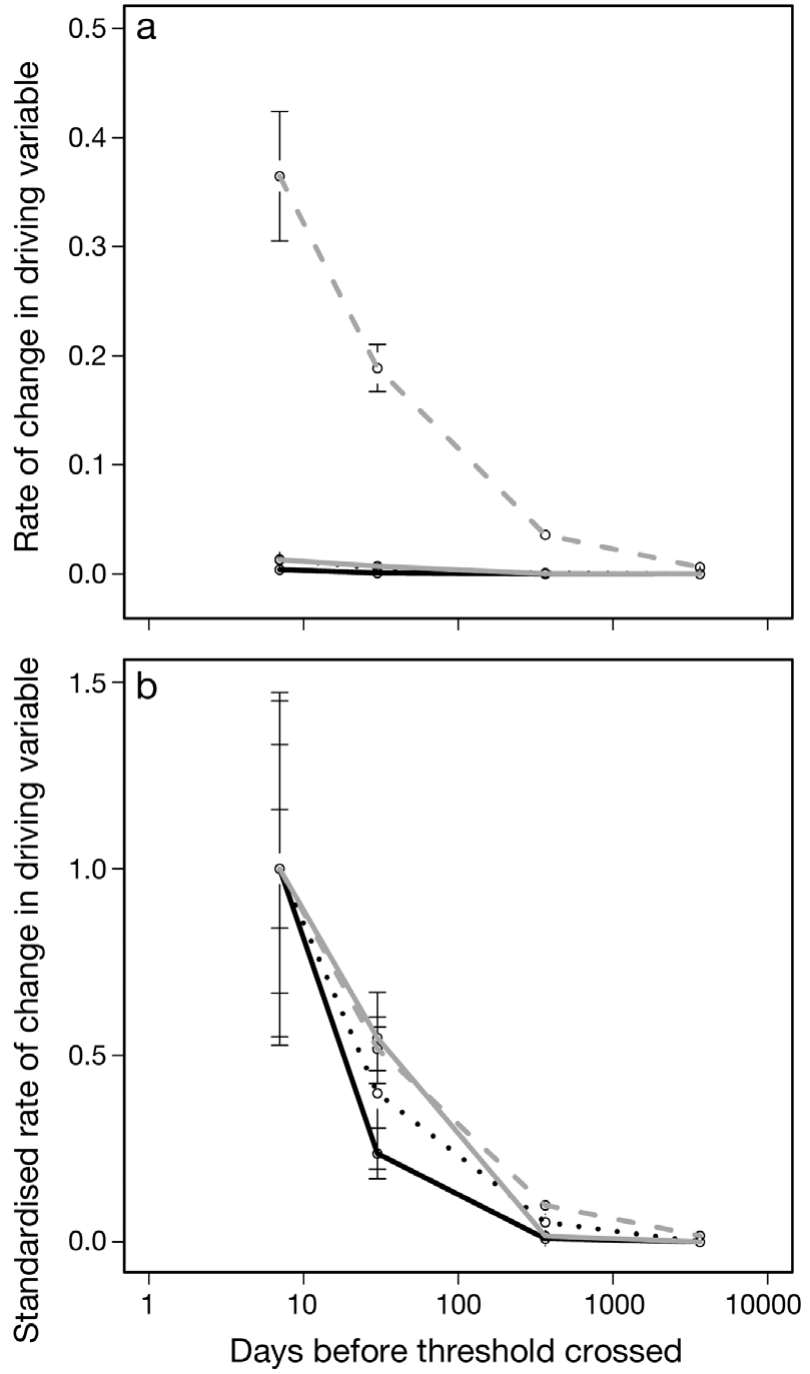

Fig. 1. Evidence of increasing rates of change in days preceding a threshold crossing for 4 variables (note log scale on the $x$-axis): tidal range $\left(\mathrm{m} \mathrm{d}^{-1}\right.$; black solid line), salinity $\left(\mathrm{g} \mathrm{l}^{-1} \mathrm{~d}^{-1}\right.$; grey dashed line), depth ( $\mathrm{m} \mathrm{d}^{-1}$; black dotted line) and water level A (m AHD d ${ }^{-1}$; grey solid line), given as (a) raw values and (b) scaled to the maximum value in each variable. Error bars are $\mathrm{SE}$

\section{Logistic regression}

Logistic regression of the predictive variables to determine the shape of the transition was done for the tidal range, water level A and maximum number of days since flow thresholds. Regression analysis was not undertaken for the water level B, depth or salinity thresholds. The resultant regression equations showed very sharp transitions. The regression for the water level A threshold (Fig. 2) is presented here as an example of the typical behaviour observed, with other variables (i.e. tidal range and maximum number of days since flow) showing similar and consistent patterns. 


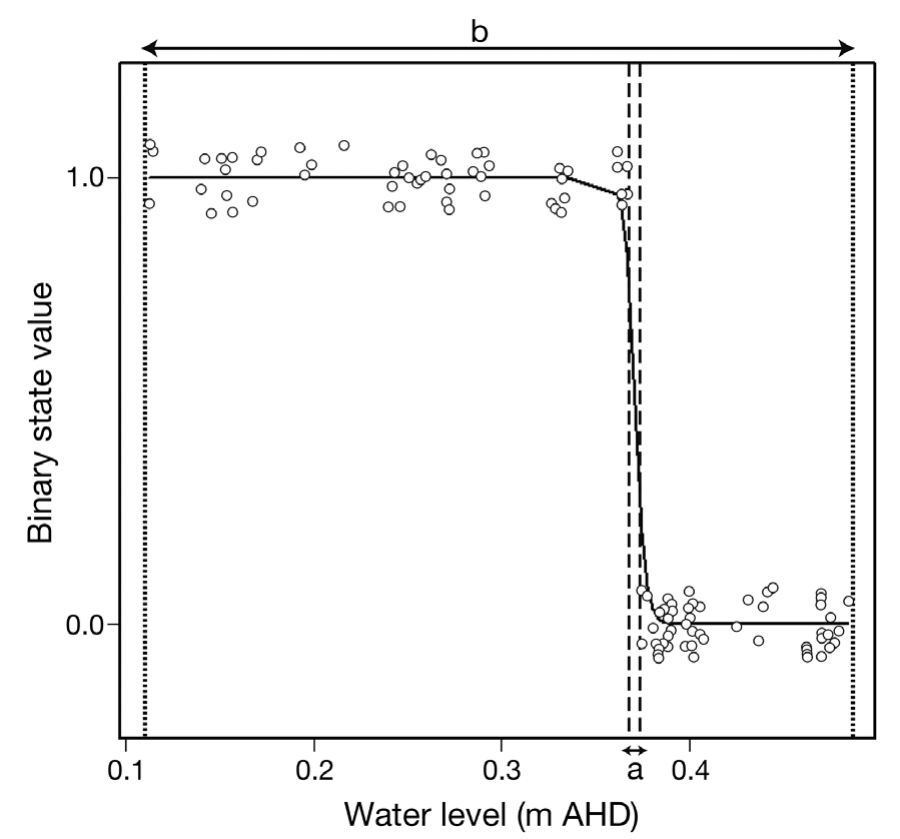

Fig. 2. Logistic regression of threshold crossings for water level A resulting in transitions in ecosystem state. Circles are the data points from the hydrodynamic modelling and have been jittered to assist in visualisation and interpretation (thus the position of identical points has been varied slightly so that all points are visible). The solid line is the line fitted to predicted values from the logistic regression. Domains ' $a$ ' (between the dashed vertical lines) and ' $b$ ' (between the dotted vertical lines) correspond to values given in Table 4 and show the smallest and largest distance across a threshold, respectively. m AHD: metres above the Australian Height Datum, which approximates sea level

The spread of values for each variable around the threshold tended to be quite continuous (i.e. there was no jump in values on either side of the threshold, suggesting a gradual change in the variable of interest), consistent with the notion that small changes in physical variables can result in large changes in biological communities. Ratios comparing the largest and smallest differences across a threshold were large for all variables, indicating inconsistencies in the size of shifts across each threshold (Table 4).

\section{Zones of transition}

In contrast to the sharp transitions identified using logistic regression, exploration of any zone of transition showed extremely wide zones around each threshold (where sufficient numbers of tran- sitions occurred for analysis). Again, the water level A threshold has been used as an example (Fig. 3), but other thresholds showed similar behaviour. For this water level threshold, $94 \%$ of all cases fell within the bounds of values that occurred in the year immediately prior to or following a transition (illustrated by domain 'b' in Fig. 2), suggesting that using presence within this wide zone of transition would not be a useful predictor of impending transition because only the most extreme cases fell outside this zone of transition and many false positives would be returned. For other thresholds, the proportion of cases falling within the zone of transition was $51 \%$ for tidal range and $68 \%$ for the maximum number of days without flow. Again, no zones were computed for the other thresholds due to the small number of transitions observed across each.

\section{A predictive model for future degraded ecosystem states}

Identifying an appropriate time lag

The strongest cross-correlations were observed between flow and hydrodynamic variables in the Coorong. All cross-correlations undertaken comparing flow and hydrodynamic variables had at least one significant time lag (Table 2). One year time lags were the strongest most commonly for flow and hydrodynamic variables (Fig. 4), indicating a close relationship between flow, water levels and salinity within the Coorong. Periodicity was also evident in the pattern of significant lags, so that 2, 3 and 4 yr lags were also statistically significant for a high proportion of the crosscorrelations investigated (Table 2).

Table 4. Width of the minimum and maximum zones of transition for each threshold. Thresholds for water level B, salinity and depth were not crossed enough times to justify logistic regression (i.e. $<3$ in each case). m AHD: metres above the Australian Height Datum, which approximates sea level

\begin{tabular}{|lccc|}
\hline Threshold & $\begin{array}{c}\text { Smallest distance } \\
\text { across a transition } \\
\text { ('a' in Fig. 2) }\end{array}$ & $\begin{array}{c}\text { Largest distance } \\
\text { across a transition } \\
\text { ('b' in Fig. 2) }\end{array}$ & Ratio b:a \\
\hline & $0.00^{\mathrm{a}}$ & 0.09 & $300.67^{\mathrm{b}}$ \\
Tidal range (m) & 74 & 577 & 7.80 \\
Maximum no. days since flow & 74 & 421 & 5.69 \\
Hypersaline (southern) basin & 0.01 & 0.37 & 44.83 \\
Marine (northern) basin & & & \\
Water level A (m AHD) & and & \\
'Size of the difference in tidal range was below the precision of the hydrody- \\
namic model, so it is presented as 0.00 \\
balue of 'a' was below the precision of the hydrodynamic model, so this value \\
should be interpreted with caution, but is presented to demonstrate the size of \\
the difference in zones of transition for tidal range \\
\hline
\end{tabular}




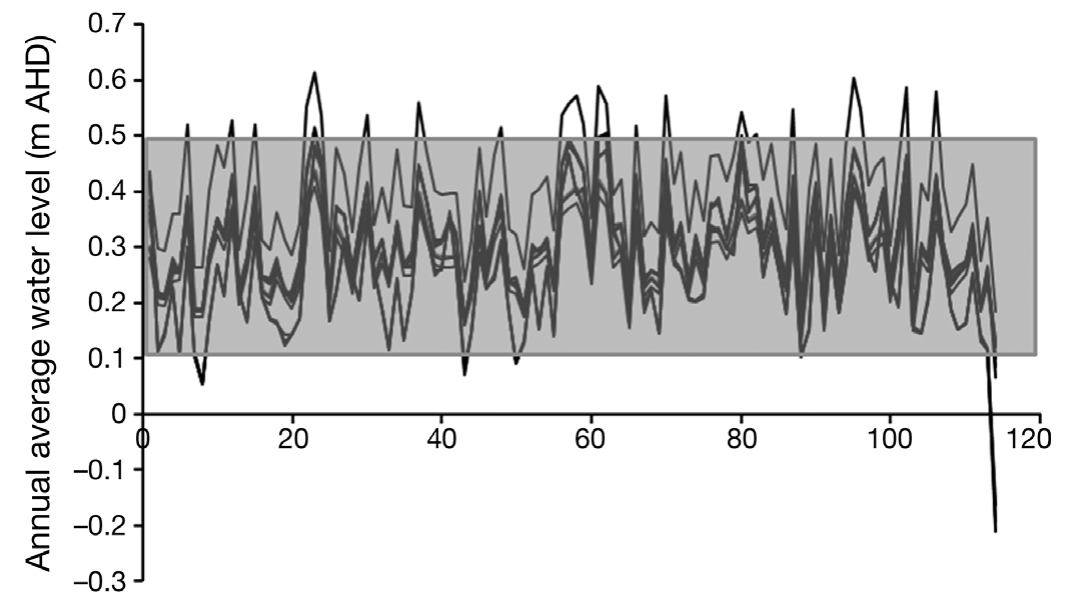

Year of simulation

Fig. 3. Extent of the zone of transition for the water level A threshold. Each line predicts a trajectory of predicted average annual water levels for one site, based on a simulation of historical conditions within the Coorong over $114 \mathrm{yr}$ for each of 12 focal sites in the Coorong. The shaded area indicates the extent of the zone of transition that encompasses the highest water level in the year prior to the threshold being crossed and the lowest water level in the year following the threshold crossing (or vice versa). m AHD: metres above the Australian Height Datum, which approximates sea level. Note that water levels below $0 \mathrm{~m}$ AHD occurred for 3 sites at the end of the simulation

ally the most common significant crosscorrelation (Table 2), but all lags between 1 and 8 yr showed similar numbers of significant correlations. The strongest correlations occurred for longer time lags, with lags of more than $5 \mathrm{yr}$ being most commonly the strongest (Fig. 4).

Modelling future degraded ecosystem states

The 1 and 3 yr intervals were used as the time lags over which to model future degraded ecosystem states in the Coorong. Both models were relatively simple, with only 1 or 2 predictive variables needed (Fig. 5).

The 1 yr model correctly predicted all degraded site-years (Fig. 5a), but also predicted that $15 \%$ of healthy site-years would be degraded, to give an overall misclassification rate of $14 \%$. Significant predictive variables were the average daily flow volume over the barrages

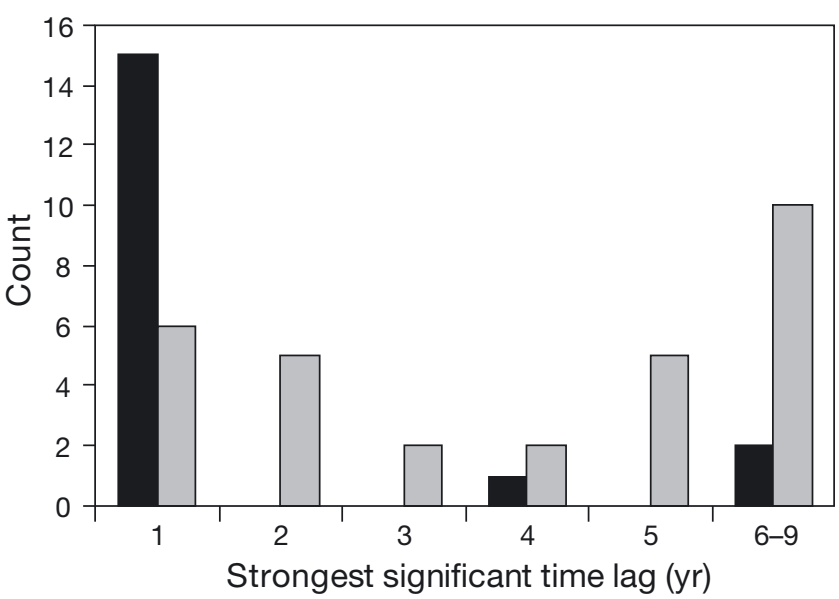

Fig. 4. Cross-correlation histograms showing the frequency of the strongest significant correlations for flow versus hydrodynamic variables (black bars), and biological versus flow, hydrodynamic and biological variables (grey bars). Refer to 'Methods: Identifying an appropriate time lag' for additional information on the variables used

Relationships amongst flow, hydrodynamic and biological variables were weaker. There were fewer significant cross-correlations observed (Table 2) and there was less consistency in the lags that tended to be significantly correlated among variables (Fig. 4). Interestingly, biological variables were generally more strongly cross-correlated with other biological variables than with either flow or hydrodynamic variables. A 2 yr time lag was margin- (adjusted for the distance of the relevant site along the Coorong) and the maximum depth of water reached for the year. Thus we conclude that degradation next year could be predicted by monitoring daily flow and water depth.

For the 3 yr model (Fig. 5b), there was a single predictive variable, average annual South Lagoon salinity. Again, all degraded site-years were correctly classified. Here, $21 \%$ of healthy site-years were predicted to be degraded, for an overall misclassification rate of $15 \%$. Thus we conclude that degradation 3 yr hence could be predicted by monitoring South Lagoon salinity now.

\section{DISCUSSION}

Several different approaches were used here to explore the behaviour of physico-chemical variables around thresholds with a view to identifying any mechanism for predicting upcoming ecological transitions. These methods included investigating how rates of change varied as thresholds approached, identification of a zone of transition around each threshold, and comparison with trends in environmental forcing factors. Rates of change and variability in rates of change increased near transitions but not within a time frame to assist managers. The increase happened late during the time series (see Fig. 1, note the log scale for time) and thus there was not much early-warning capacity. It 
a
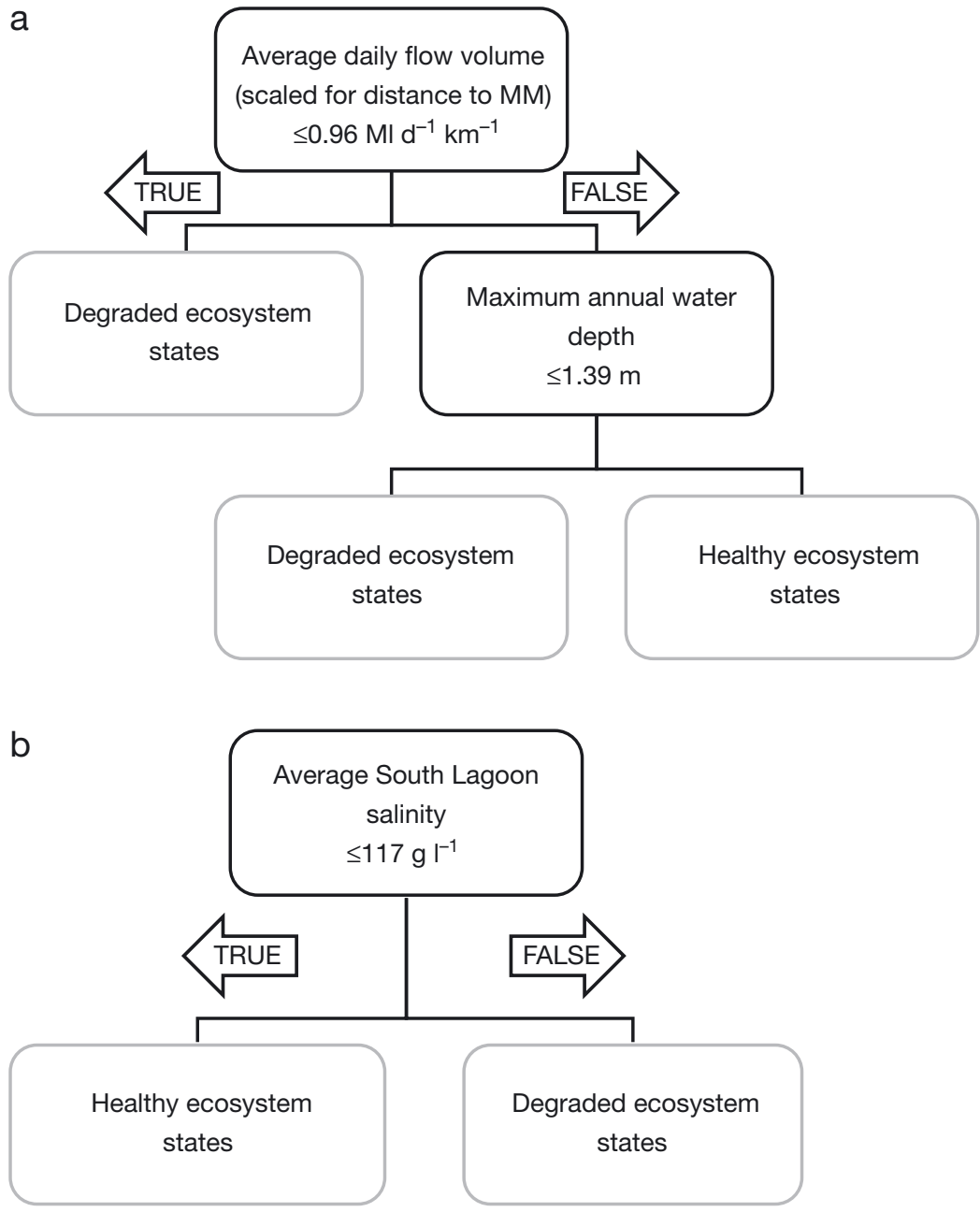

Fig. 5. Classification and regression tree models to predict future degraded ecosystem states for (a) $1 \mathrm{yr}$ in advance and (b) $3 \mathrm{yr}$ in advance. Each is presented as a logic tree, which can be followed to identify whether degraded ecosystem states are likely in either 1 or 3 yr time. Each decision box (black outline) contains a splitting parameter and its threshold value. The arrows indicate the direction the tree should be followed when the statement is true versus false. When a terminal node box (grey outline) is reached, the future presence of either degraded or healthy ecosystem states has been identified. Degraded ecosystem states (5 of 8) are defined as those that occur when no freshwater barrage flows have occurred in $339 \mathrm{~d}$; healthy ecosystem states (3 of 8) are all others. MM: Murray Mouth

is likely that by the time managers were aware that rates of change were increasing, the threshold would have been crossed and thus intervention would no longer be possible.

Attempts to identify a zone of transition were also of limited value in predicting upcoming ecological transitions. Logistic regression did not give a realistic idea of the fuzziness of thresholds, which were extremely sharp, thus giving rise to an impression of (false) precision. The crispness of these transitions is likely to be an artefact of the CART methodology used to produce the model, and such apparent precision may not be biolog- ically meaningful. In an attempt to remedy this artificial crispness, we investigated the range of values that fell within a past observed zone of transition. This approach had the other problem: that zone was too broad to be of use. In our case, 51 to $94 \%$ of occasions fell within the zone in which transitions were possible in the scenario investigated, yet obviously did not always result in transitions (see Table 3).

Thus the question remains of how to 'fuzzify' the artificially sharp transitions arising from the CART methodology without producing zones too broad to be of use. Fuzzy logic techniques have been applied in some ecological systems (Prato 2005) in a manner that may be of use for this application. Field experiments focused on how ecosystems respond in practice are also likely to be necessary to define real ranges of transition. These experiments should be based on specific hypotheses regarding the mechanisms by which components of the ecosystem respond. For example, the levels of treatments used should be set as a range-finding exercise to determine whether the thresholds are crisp or fuzzy. Such an experiment would use the observed distribution of values around the analytically-derived threshold (e.g. from CART analyses) to set the upper and lower bounds of treatment levels (Petraitis 1998). Thus the results of such an experiment would directly relate to the crispness of thresholds in practice.

In the past, efforts to predict approaching ecological transitions using thresholds have focused upon characteristics such as changes in variances, critical slowing down, changes in return rates or skewness (Carpenter \& Brock 2006, Carpenter et al. 2008, Dakos et al. 2008, Guttal \& Jayaprakash 2008). These have largely been detected using deterministic models describing system behaviour in various response variables. The utility of these characteristics for guiding management of complex ecosystems has, in most cases, yet to be determined. The detection of some of these characteristics requires significant data resources, for example complete and lengthy time series, and not all of the leading indicators seem to become apparent in a time frame that would allow management intervention (e.g. see simu- 
lations in Carpenter et al. 2008). Our alternative approach used thresholds derived from a statistical model, and focused on the behaviour of thresholds derived from predictive variables (as there is no time series of values associated with ecosystem states; they are either present or absent). Here we used descriptions of the past to infer the future and so focused on a nested set of rates of change, logistic regression, environmental forcing and zones of transition. However, these approaches also proved of limited use for management with the results either too precise or not precise enough to be useful, or where the early warning comes too late.

Instead, more value was seen in using lagged environmental variables to identify changes toward degraded states. It is theoretically possible to construct models of these leading indicators using different lengths of lag. Models were developed at both 1 and $3 \mathrm{yr}$ lags to investigate differences in the drivers of degraded ecosystem states at different points in time, as well as to provide managers with ongoing predictions of the likely future condition of the region. Both resulted in quite good levels of prediction, given the inherent variability of the system and the time lags involved (particularly for $3 \mathrm{yr}$ in advance). A $3 \mathrm{yr}$ advance model, in particular, would give sufficient warning to managers to intervene in the system to prevent future degradation (see below).

The choice of time lag based on cross-correlations for these predictive models was not clear-cut. Particularly for relationships involving biological variables, many lags had numerous significant correlations. Therefore, we based our choice of 1 and 3 yr lags on the practicality of using the resultant models for management of the system. Three years gives good opportunity for managers to act. In a large system like the Murray-Darling Basin, it would realistically take at least 1 yr to deliver additional environmental water by the time the need for that water had been established, politically sanctioned and then the water sourced and actually diverted to the estuary. Three years would give managers at least $1 \mathrm{yr}$ to source the water and $1 \mathrm{yr}$ for the effects of that water to begin to be apparent. This would then allow managers to use the 1 yr model to see if the intervention had been successful. There would be much less opportunity to intervene with only a 1 yr lag, but this model could highlight any imminent need for emergency actions. Time frames longer than 3 yr are outside (Australian) political time frames and many management planning cycles, so are not likely to be practical for informing the management of the site, because it is unlikely that water could be reliably secured that far in advance. In addition, weather within the MurrayDarling Basin is so variable that we expect the predictive capacity of the models would diminish as the lag involved increased, and it would be more difficult to justify that the water be required in the long run. The wisdom of allowing ecological modelling to be driven by political time periods is debatable, and it could be argued that management, in particular, should be driven by ecological time frames, rather than vice versa. We do not pretend to have a solution for this philosophical question, but better understanding the significant time lags within an ecological system should contribute to future debate on the issue.

Thus the 2 models were derived quite deliberately. We wanted to investigate the consistency of the predictors of future degraded ecosystem states over time. The 2 models we derived for the different time lags gave different thresholds and had different predictors (i.e. the physico-chemical variables involved) to focus upon. Thus the choice of indicator of future degradation is specific to the time frame involved. Managers should not expect modelling (or any other approach to natural resource management) to provide a 'silver bullet' that will cover all situations. Instead they need to look at a range of threshold values to fit specific purposes and then use them appropriately. It would also be possible to combine the use of the different models in clever ways to allow for different decisions to be made. For example, the 3 yr model could be used to predict future degraded states, the system could be managed accordingly and then the 1 yr model (with its different thresholds) implemented to test whether the intervention that was imposed had indeed succeeded in avoiding those conditions associated with a high likelihood of future degraded states, before they occurred.

Both the 1 and 3 yr models tended to err on the side of conservatism, with all observed misclassifications consisting of site-years that supported healthy ecosystem states being predicted to be degraded (Type I error). A moderate bias for such conservatism is almost certainly preferable to failing to predict degradation when it does occur (Type II error), because it allows managers the opportunity to intervene so as to prevent irreparable harm to the ecosystem (Fairweather 1991). Unnecessary intervention in up to $15 \%$ of cases is not likely to be unreasonable, given the inherent climatic and flow variability within the system and the time frame of up to $3 \mathrm{yr}$ in advance.

Thus modelling specifically for upcoming degradation did identify useful trigger levels in a suitable time frame, so this approach could be used to inform future management (e.g. by using the leading indicator of average South Lagoon salinity for degradation $3 \mathrm{yr}$ hence as a trigger for environmental flows or for other restoration/rehabilitation actions), but it does not provide clues about as yet unknown thresholds in the system (e.g. transition to a novel but overly degraded state) as the threshold dynamics options were looking 
to explore. Thus we can never know when we are approaching a new threshold using this method.

There is another caveat to this approach. In many cases, we have not yet tested the predictions from the models (Lester \& Fairweather 2009a). The outcomes that are predicted should be contrasted against what observations occur over the fullness of time, but we also feel that targeted experiments might be possible to speed up our understanding of how these models translate in practice. Experiments could attempt to force specific predictive variables over thresholds and then observe whether the predicted state changes occurred. This could be actively done on a small scale, or could be undertaken more passively: following the development of a stated hypothesis, a wait-and-see approach could be adopted and the behaviour of the system then observed. Additional water will either be available for the system or not, and in either case specific hypotheses could be developed based on both the 1 and 3 yr models and compared with observations. We would also suggest the use of time-series analysis to routinely explore possible lags for both auto-correlation (within an environmental variable) and crosscorrelations (amongst sets of variables) as a means to better understand the overall system behaviour for predictive purposes, as done here.

The present study illustrates the nature of threshold dynamics for one Australian estuary. It does, however, raise several points of interest for other estuarine and marine ecosystems, should these patterns prove applicable more broadly. Different estuaries will show other characteristics and different behaviour, and so need to be modelled by our general approach to identify ecosystem states in terms of biota and the environmental transitions between them. Here the ecological response model we developed was built at 2 distinct time frames (i.e. seasonally and annually) with 12 sites distributed along the Coorong. The modelling approach, however, is not constrained to these, and spatio-temporal scales can be changed to reflect the available data. Despite the spatio-temporal resolution included here, there was a general lack of portents identified for changes in ecosystem states in the estuary based on threshold dynamics. This suggests that focus should be placed on those aspects of threshold dynamics that have been shown to be able to potentially predict changes in ecosystem state (e.g. identified via direct modelling); these should be tested with monitoring and then management should be directed accordingly.

It is clear that concern about climate change will put more pressure upon water resources into the future, particularly for semi-arid estuaries. An essentially synthetic approach can be seen being actively developed in a few studies to date from across the globe (e.g. see Anderson et al. 2006, Powell 2008, Kim \& Montagna
2009, Lester et al. 2009, Zweig \& Kitchens 2009) to fulfil a need that water resource managers shall feel keenly over the years to come. This synthesis entails an ability to realistically infer what the future climate is likely to be in specific locations, translate how that might diminish water flows but increase demands for irrigation and other extractive uses, and then foresee the ecological consequences of such changes. Given this growing need, models that are able to predict future ecological degradation in a suitable time frame, with reasonable predictive accuracy, will be a significant and useful resource for estuarine management.

In conclusion, our main recommendations are that exploring threshold dynamics may not be the best method for predicting upcoming changes in ecosystem states with a view to preventing ecological degradation through active management. Instead we may need to identify leading indicators of likely future change by specifically modelling for known degraded states in the future and thus identifying usable thresholds to trigger management action in the region. However, a mechanism for predicting unknown transitions in the future remains a key knowledge gap.

Acknowledgements. The ecosystem response model used here was developed as part of the CLLAMMecology Research Cluster supported by the CSIRO Flagship Collaboration Fund and represents a collaboration between CSIRO, the University of Adelaide, Flinders University and SARDI Aquatic Sciences. Thus we thank all participants in that program, in particular the many researchers who provided data sets for the initial modelling. Significant contributions to our conceptualisation of this modelling were also made by S. Lake, G. Likens, P. Petraitis, G. Quinn, D. Walker and I. Webster. We thank R. Langley and B. Hamilton for sterling research assistance with this modelling and data presentation, J. Davis from the Murray-Darling Basin Authority for providing the flow sequence and I. Webster for providing the hydrodynamic simulations used herein. We also thank 3 anonymous referees for their useful comments.

\section{LITERATURE CITED}

Andersen T, Carstensen J, Hernández-García E, Duarte CM (2009) Ecological thresholds and regime shifts: approaches to identification. Trends Ecol Evol 24:49-57

Anderson KE, Paul AJ, McCauley E, Jackson LJ, Post JR, Nisbet RM (2006) Instream flow needs in streams and rivers: the importance of understanding ecological dynamics. Front Ecol Environ 4:309-318

BOM (Bureau of Meteorology) (2009) ENSO wrap-up. Australian Bureau of Meteorology, Melbourne, available at www.bom.gov.au/climate/enso

Breiman L, Freidman JH, Olshen RA, Stone CJ (1984) Classification and regression trees. Wadsworth International Group, Belmont, CA

Brookes JD, Lamontagne S, Aldridge KT, Benger S and others (2009) An ecosystem assessment framework to guide management of the Coorong. Final report of the CLLAMMecology Research Cluster. CSIRO: Water for a Healthy Coun- 
try National Research Flagship, Canberra, available at www.clw.csiro.au/publications/waterforahealthycountry/ cllam/

Carpenter SR, Brock WA (2006) Rising variance: a leading indicator of ecological transition. Ecol Lett 9:308-315

Carpenter SR, Brock WA, Cole JJ, Kitchell JF, Pace ML (2008) Leading indicators of trophic cascades. Ecol Lett 11: 128-138

Clarke KR, Gorley RN (2006) PRIMER v6: user manual/tutorial. PRIMER-E, Plymouth

> Conley DJ, Carstensen J, Vaquer-Sunyer R, Duarte CM (2009) Ecosystem thresholds with hypoxia. Hydrobiologia 629:21-29

CSIRO (Commonwealth Scientific and Industrial Research Organisation) (2008) Water availability in the Murray. A report to the Australian Government from CSIRO MurrayDarling Basin Sustainable Yield Project. CSIRO, Canberra, available at www.csiro.au/partnerships/MDBSYReports

- Dakos V, Scheffer M, van Nes EH, Brovkin V, Petoukhov V, Held H (2008) Slowing down as an early warning signal for abrupt climate change. Proc Natl Acad Sci USA 105: $14308-14312$

> De'ath G, Fabricius KE (2000) Classification and regression trees: a powerful yet simple technique for ecological data analysis. Ecology 81:3178-3192

> Dennison WC, Lookingbill TR, Carruthers TJB, Hawkey JM, Carter SL (2007) An eye-opening approach to developing and communicating integrated environmental assessments. Front Ecol Environ 5:307-314

Denny MW, Hunt LJH, Miller LJ, Harley CDG (2009) On the prediction of extreme ecological events. Ecol Monogr 79: 397-421

deYoung B, Barange M, Beaugrand G, Harris R, Perry RI, Scheffer M, Werner F (2008) Regime shifts in marine ecosystems: detection, prediction and management. Trends Ecol Evol 23:402-409

> Fairweather PG (1991) Statistical power and design requirements for environmental monitoring. Aust J Mar Freshw Res 42:555-567

Firth D (1993) Bias reduction of maximum likelihood estimates. Biometrika 80:27-38

Folke C, Carpenter S, Walker B, Scheffer M, Elmqvist T, Gunderson L, Holling CS (2004) Regime shifts, resilience, and biodiversity in ecosystem management. Annu Rev Ecol Evol Syst 35:557-581

Fuentes M, Kittle TGF, Nychka D (2006) Sensitivity of ecological models to their climate drivers: statistical ensembles for forcing. Ecol Appl 16:99-116

Gaines SD, Denny MW (1993) The largest, smallest, highest, lowest, longest, and shortest: extremes in ecology. Ecology 74:1677-1692

> Guttal V, Jayaprakash C (2008) Changing skewness: an early warning signal of regime shifts in ecosystems. Ecol Lett 11:450-460

Kim H, Montagna PA (2009) Implications of Colorado River (Texas, USA) freshwater inflow to benthic ecosystem dynamics: a modelling study. Estuar Coast Shelf Sci 83: 491-504

Kimmel DG, Miller WD, Harding LW, Houde ED, Roman MR (2009) Estuarine ecosystem response captured using a synoptic climatology. Estuar Coasts 32:403-409

Kleinen T, Held H, Petschel-Held G (2003) The potential role of spectral properties in detecting thresholds in the Earth system: application to the thermohaline circulation. Ocean Dyn 53:53-63

Kosmidis I (2007) brglm: bias reduction in binary-response GLMs. Available at www2.warwick.ac.uk/fac/sci/statistics/ staff/research/ioannis_/kosmidis/software
Lester RE, Fairweather PG (2009a) Ecosystem states of the Coorong: an ecosystem response model. Method development and sensitivity analyses. CSIRO: Water for a Healthy Country National Research Flagship, Canberra, available at www.clw.csiro.au/publications/waterforahealthycountry/ cllam/

> Lester RE, Fairweather PG (2009b) Modelling future conditions in the degraded semi-arid estuary of Australia's largest river using ecosystem states. Estuar Coast Shelf Sci 85:1-11

Lester RE, Fairweather PG (2010) Ecosystem response modelling of the Murray Mouth and Coorong Lagoons. In: Saintilan N, Overton I (eds) Ecosystem response modelling in the Murray-Darling Basin. CSIRO Publishing, Melbourne, p 373-389

Lester RE, Webster IT, Fairweather PG, Langley RA (2009) Predicting the future ecological condition of the Coorong. Effects of management and climate change scenarios. CSIRO Water for a Healthy Country National Research Flagship, Canberra, available at www.clw.csiro.au/ publications/waterforahealthycountry/cllam/

> Lozano-Montes HM, Pitcher TJ, Haggan N (2008) Shifting environmental and cognitive baselines in the upper Gulf of California. Front Ecol Environ 6:75-80

> Mumby PJ, Hastings A, Edwards HJ (2007) Thresholds and the resilience of Caribbean coral reefs. Nature 450:98-101

Petraitis PS (1998) How can we compare the importance of ecological processes if we never ask, 'Compared to what?' In: Resetarits WJ, Bernardo J (eds) Experimental ecology: issues and perspectives. Oxford University Press, New York, NY, p 183-201

Powell JL (2008) Dead pool: Lake Powell, global warming, and the future of water in the west. University of California Press, Berkeley, CA

> Prato T (2005) A fuzzy logic approach for evaluating ecosystem sustainability. Ecol Model 187:361-368

R Core Development Team (2009) R: a language and environment for statistical computing. R Foundation for Statistical Computing, Vienna, available at www.R-project.org

Rogers DJ, Paton DC (2009) Changes in the distribution and abundance of Ruppia tuberosa in the Coorong. CSIRO: Water for a Healthy Country National Research Flagship, Canberra, available at www.clw.csiro.au/publications/ waterforahealthycountry/cllam/

Scheffer M (2009) Critical transitions in nature and society. Princeton University Press, Princeton, NJ

Scheffer M, Bascompte J, Brock WA, Brovkin V and others (2009) Early-warning signals for critical transitions. Nature 461:53-59

Steinberg D, Golovnya M (2007) CART 6.0 user's guide. Salford Systems, San Diego, CA

Suding KN, Hobbs RJ (2009) Threshold models in restoration and conservation: a developing framework. Trends Ecol Evol 24:271-279

SYSTAT (2004) SYSTAT 11 statistics manual, Vol I-III. SYSTAT Software, Richmond, CA

van de Koppel J, Herman PMJ, Thoolen P, Heip CHR (2001) Do alternate states occur in natural ecosystems? Evidence from a tidal flat. Ecology 82:3449-3461

Warwick RM, Clarke KR (1993) Increased variability as a symptom of stress in marine communities. J Exp Mar Biol Ecol 172:215-226

Webster IT (2007) Hydrodynamic modelling of the Coorong. CSIRO: Water for a Healthy Country National Research Flagship, Canberra, available at www.clw.csiro.au/ publications/waterforahealthycountry/cllam/

Zweig CL, Kitchens WM (2009) Multi-state succession in wetlands: a novel use of state and transition models. Ecology 90:1900-1909 